\title{
Subarachnoid block in prone position for giant gluteal neurofibroma
}

\begin{abstract}
Neurofibromatosis is an autosomal dominant disease of nervous system that primarily affects the development and growth of nerve cells. Hereby we present an interesting case of giant neurofibroma over the gluteal region for skin grafting. Patient was unable to lie either in supine or sitting position. In lateral position mass was encroaching the midline? We administered subarachnoid block in proneposition taking in to consideration of, regional nature of the procedure, patient's comfort and to avoid complications in changing positions of patient shortly after administering the block. We performed the block with $23 \mathrm{G}$ spinal needle by midline approach. Spinal level upto T10 was achieved within 20 mins. Patient was haemodynamically stable and surgery also got over uneventfully.
\end{abstract}

Keywords: subarachnoid block, prone position, gluteal neurofibroma

Key Messages Subarachnoid block can be performed safely in prone position. Large bore spinal needle allows the free flow of CSF against the gravity and helps in confirming the space without the need for aspiration. Incidence of PDPH is less when the patient has been placed in the prone position post-operatively.
Volume 4 Issue 2 - 2016

Patel Rajendra, Devalkar Priti, Helawar Sudha Speciality medical officer Department of anaesthesia, KEM hospital, India

\begin{abstract}
Correspondence: Sudha Helawar, Speciality medical officer Department of anaesthesia. Ist floor old building, KEM hospital, Maharashtra, Mumbai, India, Email drsushah@gmail.com
\end{abstract}

Received: April 26, 20I5 | Published: January 28, 2016

\section{Introduction}

Subarachnoid block (SAB) can be administered in positions depending on the nature of surgery, patient factors and anaesthetist's preference. Subarachnoid block under prone position has been traditionally performed for rectal, perineal and lumbar surgeries.

Performing subarachnoid block in prone position is said to be technically difficult and cardiovascular events like bradycardia and hypotension are said to be little high. In this present case subarachnoid block was planned in prone position for a debridement and skin grafting of ulcer over giant gluteal neurofibroma.

Neurofibromatosis is a genetic disorder with widespread swellings all over the body and the disease also affects organ systems. Neurofibromatosis has various anaesthetic implications.

\section{Case history}

Thirty two year old male came with a huge lump on the left buttock since 17-18 years. Patient was disabled by the weight of the lump and was bedridden since two years. An ulcer was developed over the lump since six months and patient was forced to lie in prone position since then. Mass was measuring approximately $50 * 40$ centimeters, weighing about seven to eight $\mathrm{kg}$ present over the left buttock and extending to the lumbar region (Figure 1). A diagnosis of neurofibromatosis was made from the biopsy of the ulcer.

This patient was posted for debridement of ulcer and skin grafting. After confirming the informed consent and starvation we began with the case. Prone position was given to patient. One pillow was kept one under the head and the other one under the pelvis just to make patient comfortable and keep the abdominal wall free. Since the operated site was on the lower extremities there was no need to give the typical jack knife position. Hence table was kept straight while administering the block.

Subarachnoid block was given at L3-L4 space with 23 G quinke's spinal needle (Figure 2). We had confirmed the L3-L4 interspace by free flow of CSF against the gravity (Figure 3 ). We injected $2.5 \mathrm{cc}$ of hyperbaric bupivacaine after negative aspiration of blood. T10 sensory level was attained within 20 minutes.Patient was haemodynamic ally stable. Duration of surgery was one hour. While shifting patient's sensory level was receded up to T12.

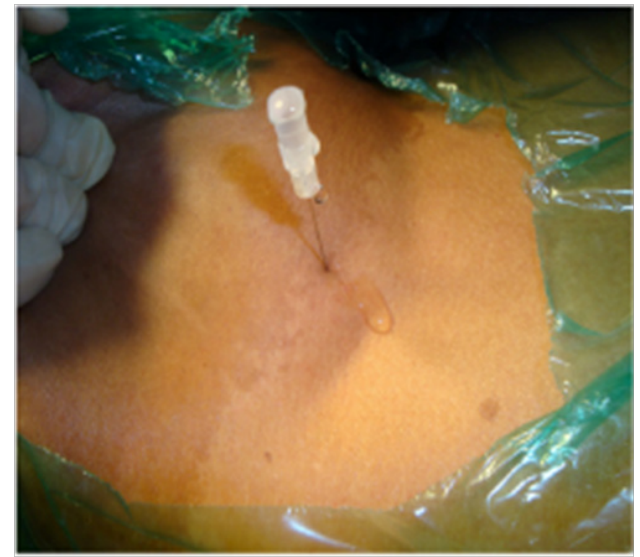

Figure I Shows free flow of CSF against gravity.

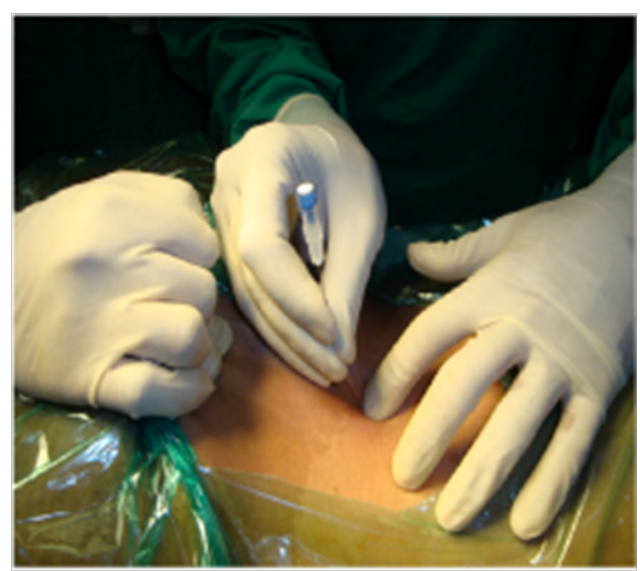

Figure 2 Shows mode of inseration of the needle. 


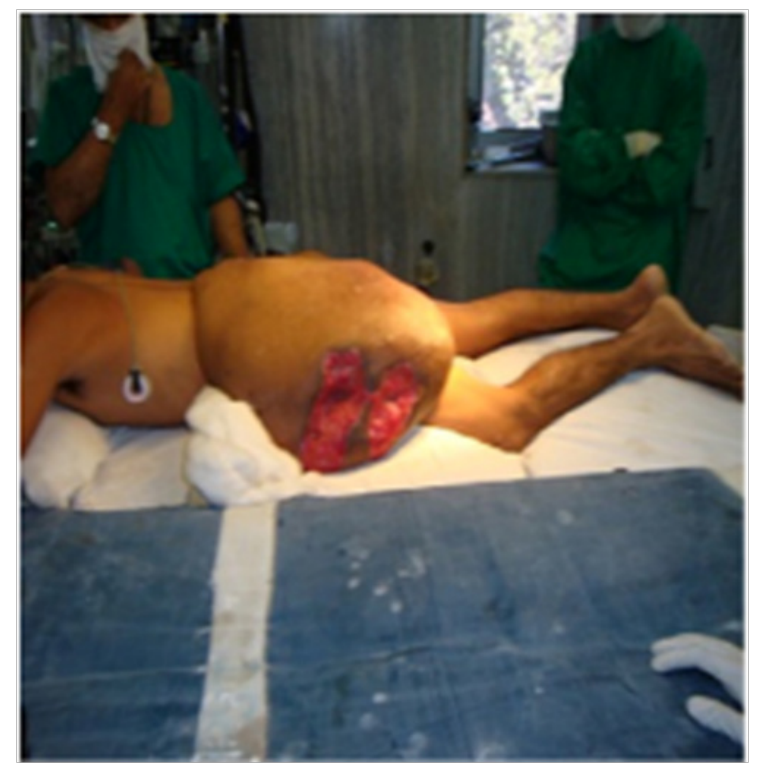

Figure 3 Shows large neurofibroma on gluteal region.

\section{Discussion}

For anaesthetizing this patient we had two options; either performs it under general anaesthesia or SAB. Our aim was to anaesthetize the patient in the prone position as same position was required for the surgery and for postoperative period. We avoided general anaesthesia because-surgery was limited to the lower extremity, patient's comfort. $\mathrm{He}$ was unable to lie in supine or lateral position. Taking into consideration the huge mass it would have been difficult to turn the patient supine for administering general anaesthesia and then again turn him prone for the surgery. Hypotension during changing the position from supine to prone could be avoided. But subarachnoid block in sitting or lateral position was also not possible. As the mass was encroaching the lumbar area in lateral position making it difficult for anatomic localization of lumbar interspinous spaces and patient was unable to sit because of the mass over gluteal region. So we opted for subarachnoid block in prone position. Subarachnoid Block in prone position has been traditionally performed for various surgeries like perineum, rectal or lumbar surgeries etc. ${ }^{1-3}$ Medhat et al. ${ }^{4}$ performed fluroscopic guided small dose spinal anaesthesia in prone position for kyphoplasty also. ${ }^{4}$ We had used 23 G Quincke's spinal needle in L3-L4 interspace. But we have realized that a large bore spinal needle could help in confirming the space by free flow of CSF against gravity. However though Richard et al. ${ }^{2}$ had used larger bore $22 \mathrm{G}$ needle by paramedian approach but Laakso and he confirmed the interspace by aspiration of CSF.

Hypobaric solution is used in prone jack knife position to specifically block the operative site which would be either perineum or rectum. Here the patient has to be operated on the gluteal region and the graft was to be taken from the opposite thigh. Hence we used $0.5 \%$ hyperbaric Bupivacaine and the table was kept straight during and after administering the block. Spinal level T10 was attained at the end of 20 mins.
Laakso et al. ${ }^{1}$ used $3 \mathrm{ml}$ of $0.5 \%$ bupivacaine and observed that on an average level attained was $\mathrm{T} 5$ for prone jack knife position and T6 for horizontal side position. ${ }^{1}$ Luiz et al. ${ }^{3}$ used $0.15 \%$ hypobaric bupivacaine. Sensory block attained was on an average T12 and only three patients presented with Motor block 3 .

Even without anaesthesia it has been demonstrated that there is $20 \%$ reduction in cardiac index in prone jack knife position due to compression of the Inferior vena cava and increase in venous pooling in the lower extremities. Laakso et al. ${ }^{1}$ had observed the mean decrease in systolic BP and bradycardia was more in prone jack knife position.

In our case the final level achieved was T10, so by avoiding turning the patient from supine to prone position for surgery we could avoid high spinal blockade. Our patient was haemodynamically stable intraoperatively and postoperatively and did not had any cardiovascular complications. During prone position the incidence of PDPH is very low.

Hence patients are asked to lie in prone position after dural puncture. ${ }^{5}$ It was observed that negative epidural pressure results in escape of CSF if lumbar spine is flexed and does not occur during extension of spine.

Our patient was lying in prone position for seven days post op and did not experience PDPH. Laasko and Richard et al. ${ }^{1,2}$ also reported very low incidence of backache and PDPH.

Our patient was awake throughout the surgery and could position himself. So the complications related to prone position under GA such as injury to eyes, pressure points, peripheral nerves, cervical spine and occlusion of carotid or vertebral artery could be avoided.

\section{Conclusion}

Hence subarachnoid block can be attempted in prone position with minimal complications. We have to be cautious about dosage of the drug, level attained and haemodynamic parameters. In our case we decided to give subarachnoid block in prone position as it was required for the surgery and patient was comfortable only in prone position.

\section{References}

1. Laakso E, Pitkänen M, Kyttä J, et al. Knee chest vs. horizontal side position of spinal anesthesia in patients undergoing surgery. $\mathrm{Br} J$ Anaesth. 1997;79(5):609-611.

2. Richard W, Charles W. Upp. The prone for the administration of spinal analgesia. Anesthesia analgesia 42. 1963.

3. Luiz EI, Marildo AG, Jose AC, et al. MV Restricted dorsal spinal anesthesia for ambulatory anorectal surgery. A pilot study. Revista Brasiliera. 1993;54(6):774-780.

4. Medhat H, Ellen G. Vance guided small dose spinal anesthesia for Kyphoplasty. Anesthesia analgesia. 1992

5. Robert JB. Technique to avoid spinal-tap headache. J Am Med Assoc. $1958 ; 168(3): 261-263$ 